\title{
Tracklet Reidentification in Crowded Scenes Using Bag of Spatio-temporal Histograms of Oriented Gradients
}

\author{
Michał Lewandowski ${ }^{1}$, Damien Simonnet ${ }^{1}$, Dimitrios Makris ${ }^{1}$, \\ Sergio A. Velastin ${ }^{1,2}$, and James Orwell ${ }^{1}$ \\ 1 Digital Imaging Research Centre, Kingston University, UK \\ 2 Department of Informatic Engineering, Universidad de Santiago de Chile, Chile \\ m. lewandowski@kingston.ac.uk
}

\begin{abstract}
A novel tracklet association framework is introduced to perform robust online re-identification of pedestrians in crowded scenes recorded by a single camera. Recent advances in multi-target tracking allow the generation of longer tracks, but problems of fragmentation and identity switching remain, due to occlusions and interactions between subjects. To address these issues, a discriminative and efficient descriptor is proposed to represent a tracklet as a bag of independent motion signatures using spatio-temporal histograms of oriented gradients. Due to the significant temporal variations of these features, they are generated only at automatically identified key poses that capture the essence of its appearance and motion. As a consequence, the re-identification involves only the most appropriate features in the bag at given time. The superiority of the methodology is demonstrated on two publicly available datasets achieving accuracy over $90 \%$ of the first rank tracklet associations.
\end{abstract}

Keywords: multi-target tracking, tracklet association, visual surveillance, histogram of oriented gradients, computer vision.

\section{Introduction}

Multi-target tracking in real crowded scenes is a fundamental problem for many vision applications, and especially for visual surveillance. The performance of pedestrian tracking algorithms has been improving to reach the point where generated tracks are increasingly reliable over longer period of time. As a consequence, the research community has devoted more attention to tracking in dense crowds.

For instance, Nevatia et al [15] propose an online learning framework to learn nonlinear motion patterns as well as robust appearance models based on estimated entry/exit points. Alternatively, Kuo el al [6] suggest a robust association using appearance based affinity measure by jointly solving ranking and classification of associations using online weight learning for weak classifiers trained previously offline. In turn, Suna et al [12] formulate the problem as maximum weight matching in a bipartite graph, whose solution is learned through a structured support vector machine. In Song et al [11], a system which simultaneously performs the scene-semantic-learning and tracking is presented and makes them supplement to each other, whereas Bo Yang et al [16] propose to learn using Conditional Random Fields and subsequently progressively associating detection responses into long tracks. 
Another line of work follows the standard tracking-by-detection procedure. While Benfold et al [2] combine detections with a Markov Chain Monte Carlo Data Association and a KLT tracker, Andriyenko et al [1] present multi-target tracking as the minimization of a continuous energy function. On the other hand, Simonnet et al [9] extend the standard framework with a hierarchy of Bayesian local predictions using a dynamic occlusion map to supplement the responses from detector. Finally, Song et al [10] introduce a stochastic graph with an evolution step that considers the statistical properties of individual tracklets, as well as the statistics of the targets along each proposed long-term track to perform association.

However, long and frequent occlusions as well as interactions between targets are still challenging, especially for tracking-by-detection approaches, and result in the generation of highly fragmented tracks, hence called 'tracklets', with frequent identity switches between them. To overcome this problem, we propose a novel tracklet association process which is formulated as a re-identification process based on the observation that, for any given pedestrian, the appearances of tracklets (e.g. before and after an occlusion) tends to be similar. As a consequence, an discriminative and efficient tracklet descriptor can be used to rank candidates and select those that correspond to the same pedestrian. In most of typical visual surveillance scenarios, even tracklets corresponding to the same individual still differ significantly, in terms of duration and orientation with respect to the camera. Moreover, they may consist of a number of walking cycle repetitions which in turn may performed at various speeds. Hence, it is extremely difficult to encapsulate all these variabilities in a single global tracklet descriptor. Therefore, we propose to represent a tracklet as a bag of local independent spatio-temporal descriptors which express the unique appearance properties of the motion, over relatively short periods of time around 'key poses'. This is achieved by first splitting trajectories of individuals into approximately linear segments based on motion direction and speed. Then, the motion signatures are extracted by analyzing periodicity of such segments. Finally, the obtained signatures are used to generate a representative set of spatio-temporal features for each tracklet. This allows a re-identification process to use only the most appropriate local descriptors at any given time, and hence substantially mitigates the impact of variability in tracklet duration, speed or orientation. As a result, the introduced tracklet re-identification framework produces not only less fragmented tracks, but also introduces less 'identity-switch' type errors.

\section{Methodology}

Fig. 1 presents an overview of the proposed approach. The whole framework consists of the following stages which are discussed in subsequent sections: tracklet generation, trajectory segmentation, periodicity analysis, local descriptors generation and tracklet association.

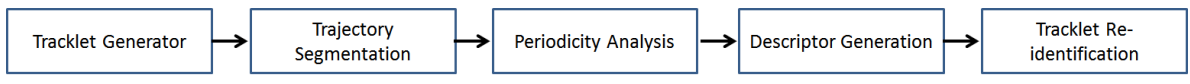

Fig. 1. An overview of tracking framework 
Tracklet Generator. The tracking of multiple targets in sets of observations is performed by an online tracklet generator. It is based on the extended tracking-by-detection principle [9]. First, pedestrians are detected globally using the HoG detector [4] followed by a local detection process using a dynamic occlusion map when no global observation is available [9]. In turn the prediction, selection of the validated measurement and update of the tracker state is carried out using the Kalman Filter [13]. Since tracklets are very likely to be correct when maintaining a spatial proximity to a single pedestrian for their duration, a tracklet classifier is incorporated to provide an indication of tracklet usability for the next stage without needing to wait until the tracklet is completed [9].It allows classification of tracklets at a given frame into three states: tentative, and reliable (when the spatio-temporal reliability condition is satisfied at each time-step up to $\Delta$ frames or after $\Delta$ frames respectively) and unreliable (at given time-step the reliability condition is not satisfied up to $\Delta$ frames). As a consequence, a tentative state stands for a tracklet which is not yet long enough to extract meaningful features for the tracklet data association step and only becomes reliable or unreliable after $\Delta$ frames. In turn, reliable tracklets are sufficiently informative to be used in the tracklet association stage, while unreliable tracklets are considered as false alarms by the system. Finally, a generated tracklet $T$ is a short sequence of appearance features with variable length $L$, i.e. $T=\left\{f_{i}\right\}_{i=1}^{L}$, which are extracted from the corresponding bounding boxes in which the target is not occluded for extended time periods or to a significant spatial extent.

Trajectory Segmentation. The extracted tracklets are split into subsequent mutually exclusive fragments (segments) so that $T=\left\{S_{s}\right\}, S_{s}=\left\{f_{i}\right\}_{i=1}^{L_{s}}, \prod_{s} S_{s} \equiv \emptyset, \sum_{s} L_{s}=$ $L$ to separate significantly different appearance observations due to motion orientation with respect to the camera. As a consequence, the periodicity analysis can be performed for each segment as well as most of identity switches can be detected. This segmentation is achieved by localizing fragments within a trajectory with an approximately constant velocity.

Initially, the trajectory for a tracklet is assembled as a temporal sequence of coordinates of pedestrians' bounding boxes (e.g. the 'centre' or 'foot' of bounding box). When analysed in a temporal domain, the trajectory is decomposed to three different motion components along vertical/horizontal direction and speed (Fig. 2). Segmentation is performed in a three level hierarchical framework. In the highest level, changes in the direction along either horizontal or vertical axis are detected. Linear segments are fitted into curve trajectory in least-squares sense using Free-Knot B-Spline approximation [8] (Fig.2). As a result, the trajectory curve is approximated by a set of linear segments which allows identification of unique peaks of sudden changes along chosen dimension (horizontal/vertical). These peaks segment the tracklet into fragments of constant direction. Afterwards this process is repeated for each segment independently in the other direction dimension (vertical or horizontal). Finally, on the lowest level each obtained segment is analysed again in terms of speed variation and when the noticable change is detected, the segment is further divided. 


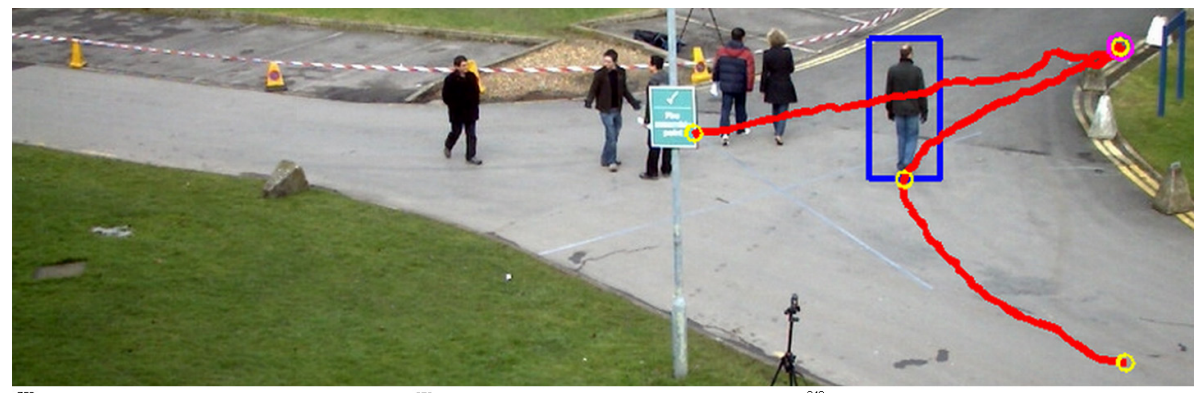

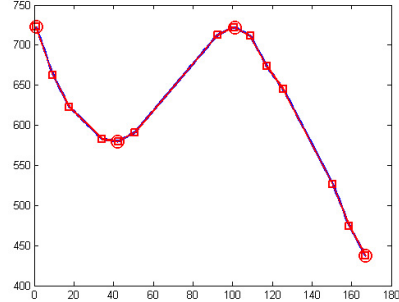

(a)

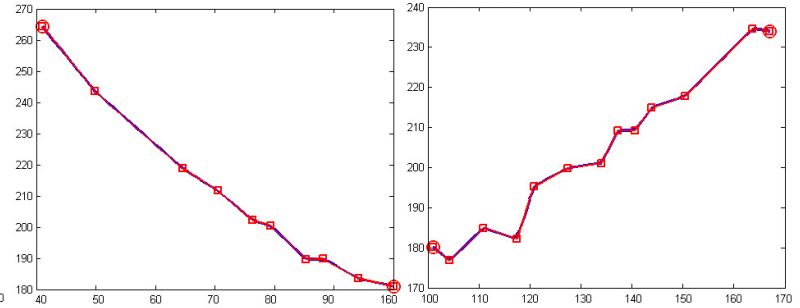

(b)

(c)

Fig. 2. Trajectory segmentation based on a horizontal or vertical direction of object position (yaxis) along the time (x-axis) where red squares correspond to borders of linear segments fitted into trajectory and red/yellow circles are peaks where the change is detected: (a) segmentation of horizontal direction into three segments, (b) and (c) segmentation of the obtained 2nd and 3rd segment respectively along vertical direction with no detected further changes

To address problem of identity switches, the tracklet $T=\left\{1, \ldots, S_{s}, \ldots\right\}$ is allowed to split into two different tracklets $T_{1}=\left\{1, \ldots, S_{s}\right\}$ and $T_{2}=\left\{S_{s+1}, \ldots\right\}$ when the estimated subsequent segments represented as vectors in the image space have opposite motion directions and an angle between them is less then a constant tolerance threshold:

$$
\arccos \left(\frac{\overrightarrow{\boldsymbol{S}_{\boldsymbol{s}}} \cdot \overrightarrow{\boldsymbol{S}_{\boldsymbol{s}+\mathbf{1}}}}{\left|\overrightarrow{\boldsymbol{S}_{\boldsymbol{s}}}\right|\left|\overrightarrow{\boldsymbol{S}_{\boldsymbol{s}+\mathbf{1}}}\right|}\right)<-A
$$

Although, this procedure cannot handle identity switches between subjects moving into roughly the same direction, it is computationally efficient and accurate for dealing with crossing identity switches. Note that in very rare cases, the abrupt turnaround of a subject may result in division of such tracklet, but such a split can generally be recovered in the tracklet re-identification process.

Periodicity Detection. After segmentation, each trajectory fragment is analysed in terms of motion periodicity to extract the key poses $f_{k}$ which repeat themselves with a constant period of $2 \pi Q^{\prime}$ where $Q^{\prime}$ is the total number of cycle repetitions within the segment. 
Initially, a square temporal self-similarity matrix is constructed for each object as it evolves in time for a given trajectory fragment $S_{s}$ [7] (Fig. 3]):

$$
\left[d_{i, j}\right]_{i, j=1 . . L_{s}}=\left[\begin{array}{ccccc}
0 & d_{1,2} & d_{2,2} & \ldots & d_{1, L_{S}} \\
d_{2,1} & 0 & d_{2,3} & \ldots & d_{2, L_{S}} \\
\ldots & \ldots & \ldots & \ldots & \ldots \\
d_{L_{S}, 1} & d_{L_{S}, 2} & d_{L_{S}, 3} & \ldots & 0
\end{array}\right] .
$$

where $d_{i, j}$ is the Euclidean distance between low level features $f$ at time instants $i, j$ like histogram of oriented gradients [4]. The autocorrelation of such self-similarity matrix is a variant of the recurrence plot which encodes different spatio-temporal properties and behaviours of dynamic systems such as periodic patterns [3]. In particular, the periodicity of the gait creates parallel diagonals in this matrix with the main diagonal of 0 corresponding to comparing frame to itself (no dissimilarity) (Fig. 3b). In turn, the temporal symmetry of gait cycles are represented by cross diagonals which are orthogonal to the main diagonal (Fig. 3r). The period of motion, i.e. the time difference between periodic patterns, can be determined by analysing the intersections between diagonal and cross diagonal lines which corresponds to the change of motion phase.

First, the 2D autocorrelation of the similarity matrix is computed using Fourier transform based on Wiener-Khinchin Theorem [14] and then smoothed by applying the Gaussian filter. As a result, local peaks along cross diagonal of autocorrelation matrix are revealed (Fig. 3b)). Then for each local extreme a diagonal 'corridor' is created of a predefined size. All extremes within this corridor with coordinates $(x, y)$ are used to fit the optimal crossed diagonal line $y=a x+b$ with parameters $\beta=[a, b]$, i.e. slope $a$ and shift $b$. This is achieved by the standard linear least square solution of an over-determined system of equations:

$$
\operatorname{argmin}_{\beta}\left\|y-\beta[x, 1]^{T}\right\|^{2}
$$

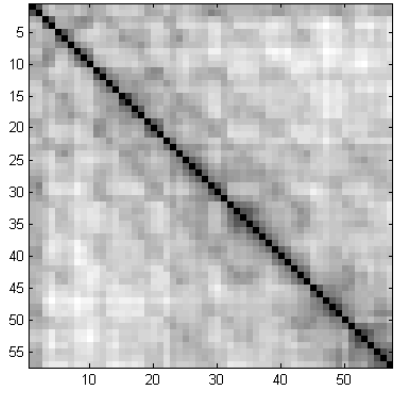

(a)

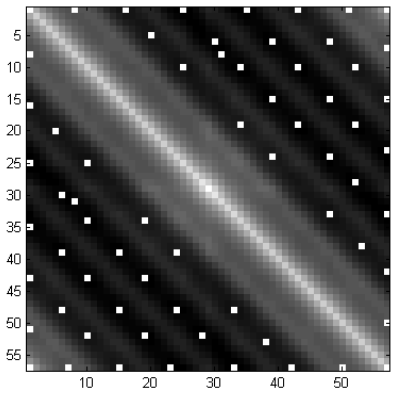

(b)

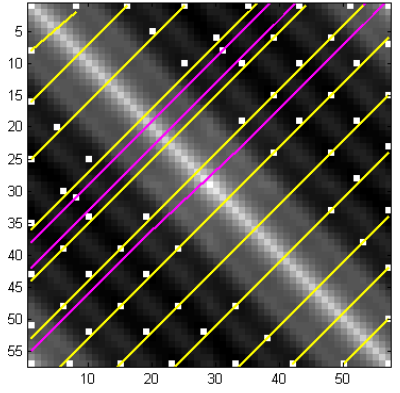

(c)

Fig. 3. (a) Temporal self-similarity matrix of a tracklet segment. (b) Autocorrelation of the selfsimilarity matrix with local peaks used to estimate parameters of cross diagonal lines. (c) Autocorrelation matrix with discarded three noisy cross diagonals (magenta) and an optimal set of cross diagonals used to determine the period of analysed motion segment (yellow). 
which minimizes the sum of squared residuals, i.e. differences between observed values and their values estimated by a model given parameters $\beta$. The approximated solution is obtained by normal equations or more numerically stable orthogonal decomposition methods such as QR or SVD decompositions.

The obtained set of $Q$ crossed diagonal lines share almost the same slope close to -1 due to temporal symmetry of the matrix and differs by shift parameters $b_{q}>b_{q-1}$ which express the intersection of the main diagonal of the autocorrelation matrix with corresponding cross diagonals. Based on recurrence plot patterns [3], each $b_{q}$ should correspond to a phase change of periodic signal, i.e. $\sin \left(b_{q}\right)=0$, so the objective is to find the best fit of periodic sine function at points of phase change, i.e. $r=[0: \pi:$ $\left.\pi * Q^{\prime}\right]$ with obtained shift parameter space by minimizing following equation:

$$
\begin{array}{r}
E_{Q^{\prime}}=\sum_{q=1}^{Q} \min \left(\left|b_{q}-r\left(Q^{\prime}\right)\right|\right), Q^{\prime}=2: Q \\
\operatorname{argmin}_{Q^{\prime}} \text { detrend }(E)
\end{array}
$$

Note that some of cross diagonals may not share the same periodicity pattern due to noisy observations (the magenta lines in Fig. 36)), therefore the whole range of phases $\pi Q^{\prime}$ needs to be analyzed to discard such noisy cross diagonals and find the best global fit. The final frame frequency $H$ between phases is given by: $H=2 * L / Q^{\prime}$, while key pose $f_{k}$ corresponds to location in time of phase change $b_{q^{\prime}}$ so that $k=\left(q^{\prime}-1\right) * H$. Fig. 4 a presents results of such minimization process with the corresponding optimal fit of sinus function into shift parameter space in Fig. $4 \mathrm{~b}$. In turn, Fig. $3 \mathrm{k}$ shows in yellow colour cross diagonals which satisfied the estimated periodicity pattern, whereas Fig. 5 illustrates some examples of detected key poses corresponding to the phase change of motion within the tracklet.

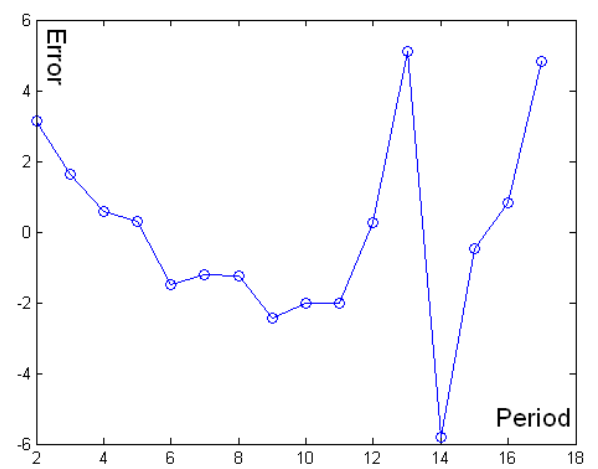

(a)

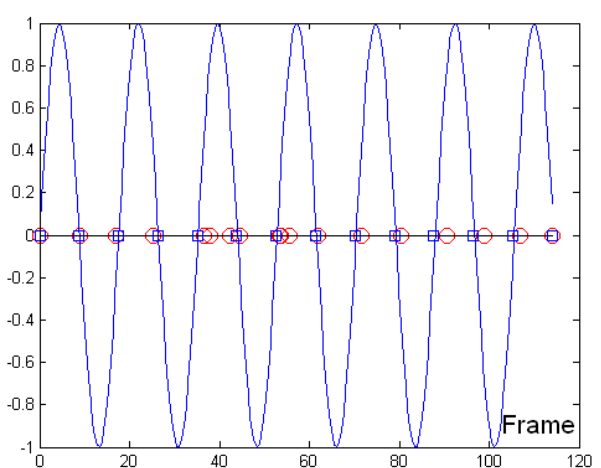

(b)

Fig. 4. (a) The detrend error of fitting periodic sine function into a sequence of shift parameters at points of phase change. (b) The best fit of periodic sine function (blue) into a sequence of shift parameters (red) at points of phase change. 

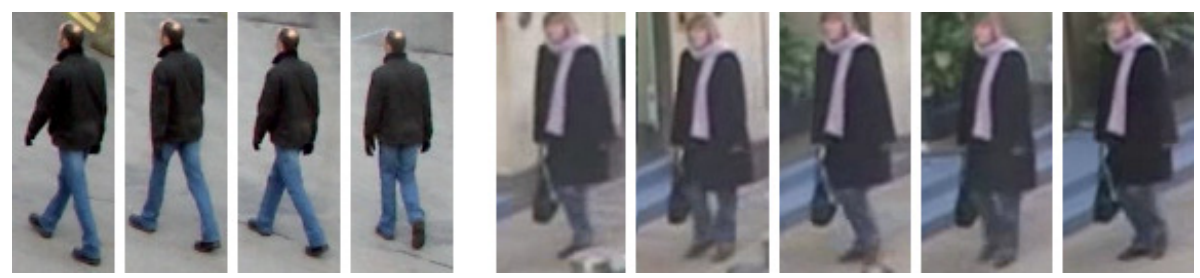

Fig. 5. Examples of detected key poses corresponding to the phase change of motion within the tracklet

Descriptor Generation. At this stage, a tracklet is represented as a bag of local and independent motion signatures $M=\left\{m_{k}\right\}, m_{k}=\left\{f_{i}\right\}_{i=k-c H / 2}^{k+c H / 2}$ of the length $c H$ where parameter $c$ controls overlapping of signatures. These signatures are generated at estimated key poses $f_{k}$ and each represented by a spatio-temporal descriptor which capture a key appearance properties of the motion over corresponding short period of time around change of motion phase. Here, we propose to adopt the histogram of oriented 3D gradients [5] as a descriptor which proves to be very effective for action recognition but to the best of our knowledge has not been yet applied for tracking. Such a histogram is extracted densely inside a bounding box based video volume of given motion signature in a very efficient way due to integral video representation of 3D gradients. Such volume is first divided into cells at different spatio-temporal scales. Then, for each cell the mean 3D gradient vector is computed and its full orientation is quantized using 3D polar binning. Afterwards, the cells are normalized and concatenated to form the histogram vector which is finally normalized over all cells. For very short tracklets or tracklets without periodic pattern, only one motion signature is generated at its centre.

Tracklet Data Association. Given a reliable 'probe' tracklet $T_{z}$, the objective is to identify and then uniquely associate the most similar subsequent tracklet among a 'gallery' set of $N_{t}$ predicted tracklets $\left(T_{z, v}\right)_{v \in \llbracket 1, N_{t} \rrbracket}$, generated during a time window $t$. This is achieved by first comparing all motion signatures of both tracklets and the best ones are ranked according to:

$$
\operatorname{rank}_{v=1 . . N_{t}}\left(W \min \left(\chi^{2}\left(M_{z}, M_{z, v}\right)\right)\right)
$$

where $\chi^{2}$ is the chi-square distance between histograms and $W$ is penalty weight expressed by: $W=\exp \left(\left\|f_{z, L}-f_{z, v, 1}\right\|\right.$ which favours candidate tracklets which are spatially closer to the probe tracklet.

\section{Evaluation}

The proposed framework was validated using two public benchmark datasets, i.e. PETS2009 [12] (Scenario S2.L1) and Oxford [2], which have been commonly used in recent multi-target tracking research. Both datasets containing typical crowded pedestrian scenes. The evaluation follows methodologies of [16 15 12] for PETS2009 
and [2]9] for Oxford to provide the fair comparison with the current state of the art approaches. The considered metrics include: recall and precision rate (showing detection performance after tracking), false alarms per frame (FA), mostly tracked (MT), mostly lost (ML) and partially tracked (PT) (the ratio of tracks with successfully tracked parts for more than $80 \%$ or less than $20 \%$ or between respectively), track fragmentation (Frag) (the number of times that a ground truth trajectory is interrupted) and id switches (IDS) (the number of times that a track changes its matched id). Note that novelty of this paper is focused on the tracklet re-identification, thus the most relevant metrics are track fragmentation and identity switches.

The tracklet generator was initialized using a few default parameters [9]. As a feature for pedestrian detection and periodicity estimation, the standard spatial histogram of oriented gradients was used [4] (8x8 pixel cells, 9 orientation bins). On the other hand, the motion signatures were represented as spatio-temporal histograms of oriented gradients [5] ( $7 \times 7 \times 2$ cells, 15 orientation bins). Motion signatures did not overlap $(c=1)$, whereas the tolerance angle for splitting tracks was set to $A=25^{\circ}$.

Tables 1 and 2 present the performance of the proposed framework in comparison to other state-of-the-art approaches. The improved performance is clearly demonstrated in the track fragmentation and identity switch metrics, which are arguably the most relevant indicators. Other metrics are still competitive with other approaches.

This is supported by obtaining almost $96 \%$ and $90 \%$ of first rank tracklet association for Oxford and PETS2009 datasets respectively (Fig. 6). This accuracy difference was expected since in the Oxford dataset motion orientation of pedestrians with respect to

Table 1. Comparison of results on Oxford sequence

\begin{tabular}{|l|c|c|c|c|c|c|c|}
\hline Method & FA & GT & MT & PT & ML & Frag & IDS \\
\hline SWT [2] & 0.190 & 201 & $0.42 \%$ & $0.46 \%$ & $12.0 \%$ & 144 & 91 \\
DTWAF [9] & 0.060 & 201 & $0.40 \%$ & $0.43 \%$ & $17.0 \%$ & 44 & 28 \\
\hline Our approach & 0.067 & 201 & $0.44 \%$ & $0.48 \%$ & $8.0 \%$ & 20 & 15 \\
\hline
\end{tabular}

Table 2. Comparison of results on PETS2009 sequence

\begin{tabular}{|l|c|c|c|c|c|c|c|c|c|}
\hline Method & Recall & Precission & FA & GT & MT & PT & ML & Frag & IDS \\
\hline Energy Min. [1] & - & - & - & 23 & $82.6 \%$ & $17.4 \%$ & $0.0 \%$ & 21 & 15 \\
PRIMPT [6] & $89.5 \%$ & $99.6 \%$ & 0.020 & 19 & $78.9 \%$ & $21.1 \%$ & $0.0 \%$ & 23 & 1 \\
NLMPRAM [15] & $91.8 \%$ & $99.0 \%$ & 0.053 & 19 & $89.5 \%$ & $10.5 \%$ & $0.0 \%$ & 9 & 0 \\
S-SVM MOT1 [12] & $97.2 \%$ & $93.7 \%$ & 0.379 & 19 & $94.7 \%$ & $5.3 \%$ & $0.0 \%$ & 19 & 4 \\
S-SVM MOT2 [12] & $96.6 \%$ & $93.4 \%$ & 0.396 & 19 & $94.7 \%$ & $5.3 \%$ & $0.0 \%$ & 26 & 5 \\
\hline Our approach & $93.3 \%$ & $99.1 \%$ & 0.025 & 19 & $89.5 \%$ & $10.5 \%$ & $0.0 \%$ & 4 & 1 \\
\hline
\end{tabular}


the camera is much more stable than in PETS2009, thus making the re-identification process more reliable. On the other hand, in the former dataset, recovery from identity switches is more of a challenge (than in the latter dataset).

Note that in contrast to our methodology, frameworks [15|12]6] require either some context information a priori or are learned online which in some scenarios may not be feasible or difficult to perform due to lack of appropriate training data. Finally, our results may be further improved when a more advanced tracker is used to generate initial tracklets.
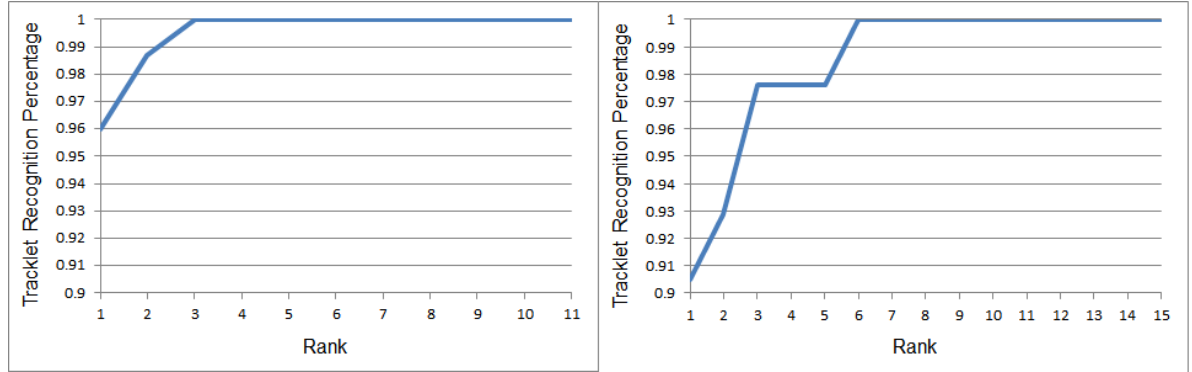

Fig. 6. Cumulative Matching Characteristic of ranking tracklets for Oxford (left) and PETS2009 (right) datasets

\section{Conclusion}

This paper has proposed a new descriptor for robust tracklet association using a bag of motion signatures expressed by spatio-temporal histograms of oriented gradients. To overcome problems of tracklet variability such as change of velocity or existence of cyclic repetitions, features in bag are generated at various time points to encapsulate key appearance and dynamic properties over short period of time. As a consequence, the ranking procedure for re-identification can take into account only the best features at given time as well as recover from badly assigned identities during tracking. The experiments have confirmed that our approach provides generally comparable performance to other methods, while being advantageous in the reduction of track fragmentation and number of identity switches, thus producing more reliable tracks.

\section{References}

1. Andriyenko, A., Schindler, K.: Multi-target tracking by continuous energy minimization. In: Proc. of CVPR (2011)

2. Benfold, B., Reid, I.: Stable Multi-Target tracking in Real-Time surveillance video. In: Proc. of CVPR (2011)

3. Cutler, R., Davis, L.S.: Robust Real-Time periodic motion detection, analysis, and applications. TPAMI (2000)

4. Dalal, N., Triggs, B.: Histograms of oriented gradients for human detection. In: Proc. of CVPR (2005) 
5. Kläser, A., Marszałek, M., Schmid, C.: A Spatio-Temporal descriptor based on 3D-gradients. In: Proc. of BMVC (2008)

6. Kuo, C.-H., Nevatia, R.: How does person identity recognition help Multi-Person tracking? In: Proc. of CVPR (2011)

7. Lele, S.: Euclidean distance matrix analysis (edma): Estimation of mean form and mean form difference. In: Mathematical Geology (1993)

8. Schütze, T., Schwetlick, H.: Constrained approximation by splines with free knots. In: BIT Numerical Mathematics (1997)

9. Simonnet, D., Lewandowski, M., Velastin, S., Orwell, J., Turkbeyler, E.: Tracking pedestrians in crowded scenes using dynamic time-warped appearance features. In: Workshop PCRA (2012)

10. Song, B., Jeng, T.-Y., Staudt, E., Roy-Chowdhury, A.K.: A stochastic graph evolution framework for robust multi-target tracking. In: Daniilidis, K., Maragos, P., Paragios, N. (eds.) ECCV 2010, Part I. LNCS, vol. 6311, pp. 605-619. Springer, Heidelberg (2010)

11. Song, X., Shao, X., Zhao, H., Cui, J., Shibasaki, R., Zha, H.: An online approach: Learningsemantic-scene-by-tracking and tracking-by-learning-semantic-scene. In: Proc. of CVPR (2010)

12. Kim, S., Kwak, S., Feyereisl, J., Han, B.: Online multi-target tracking by large margin structured learning. In: Lee, K.M., Matsushita, Y., Rehg, J.M., Hu, Z. (eds.) ACCV 2012, Part III. LNCS, vol. 7726, pp. 98-111. Springer, Heidelberg (2013)

13. Welch, G., Bishop, G.: An introduction to the kalman filter (1995)

14. Wiener, N.: Generalized harmonic analysis. Acta Mathematica (1930)

15. Yang, B., Nevatia, R.: Multi-target tracking by online learning of non-linear motion patterns and robust appearance models. In: Proc. of CVPR (2012)

16. Yang, B., Huang, C., Nevatia, R.: Learning affinities and dependencies for multi-target tracking using a crf model. In: Proc. of CVPR (2011) 